\title{
MAXIMAL SUBLATTICES OF FINITE DISTRIBUTIVE LATTICES
}

\author{
IVAN RIVAL
}

\begin{abstract}
A best possible estimate is established for the size and length of maximal proper sublattices of finite distributive lattices.
\end{abstract}

1. Introduction. Papers of H. Sharp [3] and D. Steven [4] have established the following result:

THEOREM 1. If $L$ is a finite Boolean lattice with $|L| \geqq 4$ and $M$ is a maximal proper sublattice of $L$ then (i) $|M|=\frac{3}{4}|L|$ and (ii) $l(M)=l(L)$, where $l(L)$ denotes the length of $L$.

The purpose of this note is to prove an analogous result for finite distributive lattices, as well as to provide a simple alternative proof of Theorem 1.

THEOREM 2. If $L$ is a finite distributive lattice with $|L| \geqq 3$ and $M$ is a maximal proper sublattice of $L$ then (i) $|M| \geqq \frac{2}{3}|L|$ and (ii) $l(M) \geqq l(L)-1$.

Furthermore, these inequalities are best possible in the sense that for every integer $n \geqq 1$ there is a distributive lattice $L_{n}$ with a maximal proper sublattice $M_{n}$ such that $\left|L_{n}\right|=3 n,\left|M_{n}\right|=2 n$ and $l\left(M_{n}\right)=l\left(L_{n}\right)-1$ (see Figure 1).

2. Preliminaries. Let $J(L)=\{x \in L \mid x$ join-irreducible $\}, M(L)=\{x \in L \mid x$ meet-irreducible $\}$ and for all further notation and terminology refer to [2]. Recall that in lattices of finite length every element can be expressed as the join of all join-irreducibles contained in it and dually.

The basic result we need is that characterizing maximal proper sublattices of an arbitrary finite distributive lattice [1]. In what follows we provide a new simple proof of this result.

LEMMA 1. If $L$ is a lattice of finite length and $S$ is a proper sublattice then there exist $a \in J(L), b \in M(L), a \leqq b$, such that $S \cap[a, b]=\varnothing$.

Received by the editors April 17, 1972.

AMS (MOS) subject classifications (1970). Primary 06A35; Secondary 05A15.

Key words and phrases. Distributive lattice, maximal proper sublattice.

(c) American Mathematical Society 1973 


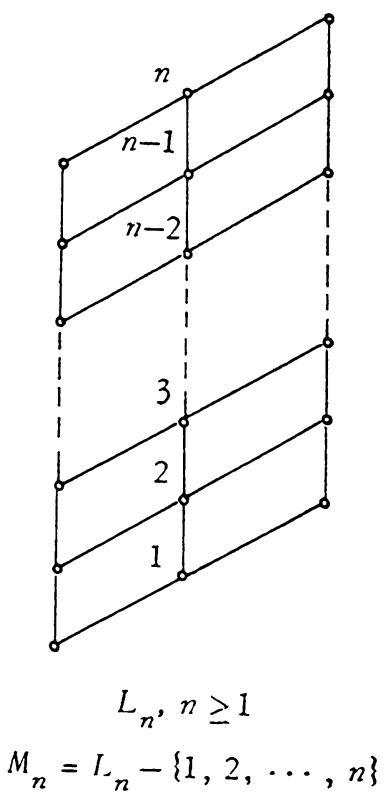

FIGURE 1

Proof. Since $S$ is a proper sublattice there exists $a \in J(L)-S$. Let $B=$ $\{b \in M(L) \mid a \leqq b\}$; clearly $B \neq \varnothing$. If for every $b \in B$ there were some $x_{b} \in S \cap[a, b]$ then $\wedge\left(x_{b} \mid b \in B\right) \in S$ since $S$ is a complete sublattice. On the other hand, $a \leqq \bigwedge\left(x_{b} \mid b \in B\right) \leqq \bigwedge B=a$, that is, $\bigwedge\left(x_{b} \mid b \in B\right)=a \notin S$, which is a contradiction. Thus, we can conclude that there is some $b \in B$ such that $S \cap[a, b]=\varnothing$.

LeMmA 2. If $L$ is a distributive lattice, $a \in J(L), b \in M(L)$, and $a \leqq b$, then $L-[a, b]$ is a sublattice of $L$.

Proof. Let us assume that there exist $x, y \in L-[a, b]$ such that $x \vee y \in[a, b]$. Since $a \in J(L)$ and $L$ is distributive we get that $a \leqq x$ or $a \leqq y$, so that either $x \in[a, b]$ or $y \in[a, b]$, contradicting our choice.

We can now give the basic characterization [1].

THEOREM 3. If $L$ is a finite distributive lattice and $M$ is a maximal proper sublattice, then there exist $a \in J(L), b \in M(L), a \leqq b$, such that (i) $L-M=$ $[a, b]$, (ii) $(a, b] \subseteq L-J(L)$ and (iii) $[a, b) \subseteq L-M(L)$.

Proof. (i) By Lemma 1 there exist $a \in J(L), b \in M(L), a \leqq b$, such that $M \cap[a, b]=\varnothing$, that is, $M \subseteq L-[a, b] \subset L$. By Lemma $2, L-[a, b]$ is a sublattice so that by the maximality of $M, M=L-[a, b]$. 
(ii) If there were some $c \in(a, b]$ such that $c \in J(L)$, then in view of Lemma $2, M=L-[a, b] \subseteq L-[c, b] \subset L$ is a chain of sublattices contradicting the maximality of $M$. (iii) follows dually.

We are now ready to prove Theorems 1 and 2 .

3. The Boolean case. Suppose $M$ is a maximal proper sublattice of the Boolean lattice $2^{n}$. Without loss of generality we may take $n \geqq 3$ in which case it must be that $0,1 \in M$. By Theorem 3 there exist $a \in J(L)$, $b \in M(L)$ such that $L-M=[a, b]$. But then $a$ must be an atom and $b$ a coatom, so that $[a, b] \cong 2^{n-2}$, and this, of course, implies that $|M|=\frac{3}{4}\left|2^{n}\right|$. Finally, if $a^{\prime}$ is the complement of $a$, then $\left[0, a^{\prime}\right] \cup\left[a^{\prime}, 1\right] \subseteq M$, since 0 , $1 \in M$ and $L-M$ is a convex sublattice of $L$. Since all maximal chains have the same order, $l(M)=l(L)$ which completes the proof of Theorem 1 .

4. The distributive case. The proof of Theorem 2 amounts essentially to an enumeration of sufficiently many joins and meets of subsets of $J(L)$ and $M(L)$, respectively.

We can certainly assume that $|L-M| \geqq 2$ so that by the maximality of $M, 0,1 \in M$ and $L-M \subseteq L-(J(L) \cap M(L))$. By Theorem 3 there exist $a \in J(L), b \in M(L), a \leqq b$, such that $L-M=[a, b]$.

Now, $L$ is finite, so in particular, for every $x \in(a, b]$ there exists $A \subseteq$ $J(L)$ such that $x=\bigvee A$. If $A \subseteq M$ then $\bigvee A \in M$ since $M$ is a finite sublattice. Therefore, $A \cap[a, b] \neq \varnothing$, and, in fact, by Theorem $3, J(L) \cap$ $[a, b]=\{a\}$, so that $a \in A$. This shows that for every $x \in(a, b]$ we can select a subset $f(x)$ in $J(L) \cap M$ such that $x=a \vee \bigvee f(x)$, where $\bigvee f(x) \in M$; in fact, $\bigvee f(x) \in M-\{0,1\}$. Moreover, it is immediate that if $x, y \in(a, b]$, $\bigvee f(x)=\bigvee f(y)$ if and only if $x=y$. Thus, we can conclude that

$$
\{\bigvee f(x) \mid x \in(a, b]\} \subseteq M-\{0,1\}
$$

and

$$
|\{\bigvee f(x) \mid x \in(a, b]\}|=|(a, b]|=|L-M|-1
$$

Dually, we get a choice function $g$ from $[a, b)$ into the set of subsets of $M(L)$ such that $y=b \wedge \wedge g(y)$, for every $y \in[a, b),\{\wedge g(y) \mid y \in[a, b)\} \subseteq$ $M-\{0,1\}$, and $|\{\wedge g(y) \mid y \in[a, b)\}|=|L-M|-1$.

We now claim that $\{\bigvee f(x) \mid x \in(a, b]\} \cap\{\wedge g(y) \mid y \in[a, b)\}=\varnothing$. In fact, suppose there were suitable $x, y \in[a, b]$ such that $\bigvee f(x)=\wedge g(y)$. Clearly, $b \geqq x=a \vee \bigvee f(x) \geqq \bigvee f(x)$ so that $\bigvee f(x)=b \wedge \bigvee f(x)=b \wedge \wedge g(y)=$ $y$, which is impossible since $\bigvee f(x) \in M$ while $y \in L-M$.

It now follows that $|L| \geqq|L-M|+|\{\bigvee f(x) \mid x \in(a, b]\}|+\mid\{\wedge g(y) \mid y \in$ $[a, b)\}|+|\{0,1\}|=3| L-M \mid$, from which (i) is an immediate consequence.

To establish (ii) take some $c \in L$ such that $b>c \geqq \bigvee f(b)$ (b covers $c$ ). If $c \in[a, b]$ then $c \geqq \bigvee f(b)$ and $c \geqq a$ together imply that $b>c \geqq a \wedge \bigvee f(b)=$ $b$, which is impossible. Thus, $c \in M$ and by the convexity of $L-M$, 
$[0, c] \subseteq M$. Finally, taking a maximal chain in $[0, c]$, adjoining to it a maximal chain in $(b, 1]$ and recalling that in distributive lattices all maximal chains have the same order, we can conclude that $l(M) \geqq l(L)-1$, completing the proof of Theorem 2.

\section{BIBLIOGRAPHY}

1. C. C. Chen, K. M. Koh and S. K. Tan, Frattini sublattices of distributive lattices (preprint).

2. G. Grätzer, Lattice theory: First concepts and distributive lattices, Freeman, San Francisco, Calif., 1971.

3. H. Sharp, Cardinality of finite topologies, J. Combinatorial Theory 5 (1968), 82-86. MR 37 \#2167.

4. D. Steven, Topology on finite sets, Amer. Math. Monthly 75 (1968), 739-741.

Department of Mathematics, University of Manitoba, Winnipeg, Manitoba, CANADA 